\title{
Numerical extrapolation method for complex conductivity of disordered metals
}

\author{
S. Kern, ${ }^{1}$ P. Neilinger, ${ }^{1,2}$ D. Manca,${ }^{1}$ J. Greguš,${ }^{1}$ S. Volkov, ${ }^{1}$ and M. Grajcar ${ }^{1,2}$ \\ ${ }^{1}$ Department of Experimental Physics, Comenius University, SK-84248 Bratislava, Slovakia \\ ${ }^{2}$ Institute of Physics, Slovak Academy of Sciences, Dúbravská cesta, Bratislava, Slovakia
}

\begin{abstract}
Recently, quantum corrections to optical conductivity of disordered metals up to the UV region were observed. Although this increase of conductivity with frequency, also called anti-Drude behaviour, should disappear at the electron collision frequency, such transition has never been observed, or described theoretically. Thus, the knowledge of optical conductivity in a wide frequency range is of great interest. It is well known that the extrapolation of complex conductivity is illposed - a solution of the analytic continuation problem is not unique for data with finite accuracy. However, we show that assuming physically appropriate properties of the searched function $\sigma(\omega)$, such as: symmetry, smoothness, and asymptotic solution for low and high frequencies, one can significantly restrict the set of solutions. We present a simple numerical method utilizing the radial basis function approximation and simulated annealing, which reasonably extrapolates the optical conductivity from visible frequency range down to the far infrared and up to the ultraviolet region. The method was compared with two widely used analytic continuation techniques and resulting extrapolation obtained on $\mathrm{MoC}$ and $\mathrm{NbN}$ thin films was checked by transmission measurement across a wide frequency range.
\end{abstract}

\section{INTRODUCTION}

Optical properties of thin films, especially superconducting metals, are currently of great interest. Knowledge of the electric response is essential for the rapidly expanding field of superconducting devices e.g. photon detectors, ${ }^{11}$ as well as for purely academic purposes, due to the presence of phenomena like weak localization. Therefore, this class of materials attracts much investigation.2 ${ }^{24}$ Measurement of optical properties in a broad frequency range is often demanding and the experimental window of devices is naturally limited. However, as it is known, the knowledge of the response function on a finite interval is sufficient to extend the domain because this function must be analytical, i.e. satisfying the Kramers-Kronig relations (KK). Unfortunately, this problem, known as analytic continuation (AC), is ill-posed - its solution is unstable and not uniquely determined when a small error is introduced into an input data set. ${ }^{5}$ In order to solve such a problem, regularization should be carried out by making use of additional information.

For example, one can presume that the studied system can be described by a certain physical model ${ }^{6 / 7} \mathrm{In}$ the case of metals, the complex conductivity is usually analysed in terms of the classical Drude-Lorentz model. This model allows to fit the conductivity of clean metals well, but it fails to describe the conductivity of disordered metals, where quantum corrections play a significant role ${ }^{8}$ and can persist up to the ultraviolet range.$^{4}$ The discussion on the applicability of this model is in the Appendix C. In contrast, there are more general, rather mathematical approaches to the AC, e.g. the Padé approximation and the singular value decomposition (SVD). However, they exhibit instability due to noise in the data, as we demonstrate in Appendix $\mathrm{C} 2$ and the regularization by physically motivated constraints is not straightforward 9 [1]
In this paper, we describe a method which allows to add constraints systematically until a reasonable extrapolation is obtained. As we demonstrate, our method is able to find the expected solution, presuming physically well justified restrictions on the searched function. Namely, that the complex conductivity i) cannot oscillate on scales smaller than the relaxation rate $\Gamma$, ii) cannot diminish at frequencies higher than $5 \Gamma$, iii) has a nonnegative real part and iv) the contribution from high frequency $(\gg \Gamma)$ transitions can be included in the "infinite frequency" dielectric constant $\epsilon_{\infty}$. This allows a model-independent determination of $\sigma(\omega)$, extending the experimental window of spectroscopic ellipsometry by making use of DC sheet resistance, both measured at room temperature. The approach is demonstrated on data measured on highly disordered metals close to the metal-insulator transition. Our simple illustrative procedure is compared to the aforementioned techniques, namely to the Drude-Lorentz data fit, Padé approx. and SVD.

\section{METHOD DESCRIPTION}

Let a set of complex values $\left\{\sigma_{e}^{\prime}\left(\omega_{i}^{e}\right)+i \sigma_{e}^{\prime \prime}\left(\omega_{i}^{e}\right)\right\}$ be the complex conductivity at discrete frequencies $\omega_{i}^{e}$, from interval $\left[\omega_{\min }^{e}, \omega_{\max }^{e}\right]$, as well as at zero frequency, $\sigma_{e}^{\prime}(0)=$ $\sigma_{d c}$, measured at temperature $T_{R}$. We start with the discretization of the $\omega$ - axis. Taking into account that the error of analytical continuation of experimental data measured with finite precision increases exponentially with distance from the known interval,, 5 we use a logarithmic scale. This also helps to avoid problems with splining square-root and logarithmic singularities with polynomial functions. We introduce a new discrete vari- 
able $x_{i}$ with values

$$
\begin{aligned}
x_{i}= & 0,1, \ldots \\
& \ldots k, \frac{1}{a} \ln \left(\hbar \omega_{0}^{e} / k_{B} T_{R}\right), \frac{1}{a} \ln \left(\hbar \omega_{1}^{e} / k_{B} T_{R}\right), \ldots \\
& \ldots, \frac{1}{a} \ln \left(\hbar \omega_{N}^{e} / k_{B} T_{R}\right), l, l+1, \ldots, n,
\end{aligned}
$$

where the number of points $k$ below the experimental window is set by a parameter $a=\ln \left(\hbar \omega_{0}^{e} / k_{B} T_{R}\right) /(k+1)$ and $l$ is the lowest integer greater than $\ln \left(\hbar \omega_{\text {max }}^{e} / k_{B} T_{R}\right)$. The discretization is obtained simply by

$$
\omega_{i}=\frac{k_{B} T_{R}}{\hbar} e^{a \cdot x_{i}} .
$$

An example of such procedure is shown in Fig. 11 where the exponential spacing of red points $\omega_{i}$ is interrupted by the experimental values (blue points) at $\omega_{i}^{e}$. Values of the real part of conductivity at the frequencies $\omega_{i}$ are denoted by $y_{i}$, i.e. $y_{i}=\sigma^{\prime}\left(\omega_{i}\right)$ and they will become the fitting parameters to be optimized. The integer $n$, determining the number of fitted points, can be estimated as the index of a reasonably high value of $\omega_{n}$, where the conductivity can be safely fixed to zero, since $\sigma(\omega \rightarrow$ $\infty) \rightarrow 0$, whereas the contributions from high energy transition, being far enough from the studied region, are included in parameter $\epsilon_{\infty}$ introduced later on. According to Fermi liquid theory, finite temperature can be taken into account by the transformation

$$
\omega \mapsto \Omega=\sqrt{\omega^{2}+\gamma(T)^{2}},
$$

where $\gamma(T)$ is of the same order of magnitude as $k_{B} T / \hbar$, often taken as $\gamma(T)=\pi k_{B} T / \hbar$ (e.g. Eq. 6.7 in Ref. 8). Equation (3) implies that for $\omega \ll \gamma(T)$, the conductivity $\sigma^{\prime}(\Omega)$ is constant and equals to $\sigma_{d c}=\sigma^{\prime}(\gamma(T))$. Thus a smooth function $y=f_{\left\{y_{i}\right\}}(x)$ can be constructed by a cubic spline using the Radial Basis Function (RBF) method ${ }^{12 / 13}$, with two boundary conditions, $y_{0}=\sigma_{d c}$ measured at room temperature and $y_{n}=0$. The first condition is satisfied by fixing the first point of the discretization to frequency $\approx \gamma\left(T_{R}\right)$, which is the reason for the presence of the temperature, at which the optical measurement was conducted, in the discretization (1). The spline proceeds in the logarithmic scale, where the distribution of centres $x_{i}$ given by equation (1) is equidistant, which is optimal for RBF method!14

From known trial function $f$, the complex conductivity is calculated as $\sigma_{\left\{y_{i}\right\}}^{\prime}(\omega)=f_{\left\{y_{i}\right\}}(x(\omega))$ and $\sigma_{\left\{y_{i}\right\}}^{\prime \prime}(\omega)=$ $\mathcal{H}\left[\sigma_{\left\{y_{i}\right\}}^{\prime}(\omega)\right]$. Here, $\mathcal{H}\left[\sigma_{\left\{y_{i}\right\}}^{\prime}(\omega)\right]$ is the Hilbert transform of $\sigma_{\left\{y_{i}\right\}}^{\prime}(\omega)$. The curve $f_{\left\{y_{i}\right\}}(x)$ is found as the best fit of its Hilbert transform $\sigma_{\left\{y_{i}\right\}}^{\prime \prime}(\omega)$ to experimental set of points $\sigma_{e}^{\prime \prime}\left(\omega_{i}^{e}\right)$ by least-squares method, minimizing the functional

$$
\begin{aligned}
\mathcal{F}\left[\sigma_{\left\{y_{i}\right\}}^{\prime}(\omega)\right] & =\sum_{\omega_{j}^{e}}\left(\sigma_{e}^{\prime \prime}\left(\omega_{j}^{e}\right)-\sigma_{\left\{y_{i}\right\}}^{\prime \prime}\left(\omega_{j}^{e}\right)\right)^{2} \\
& +\sum_{\omega_{j}^{e}}\left(\sigma_{e}^{\prime}\left(\omega_{j}^{e}\right)-\sigma_{\left\{y_{i}\right\}}^{\prime}\left(\omega_{j}^{e}\right)\right)^{2} .
\end{aligned}
$$

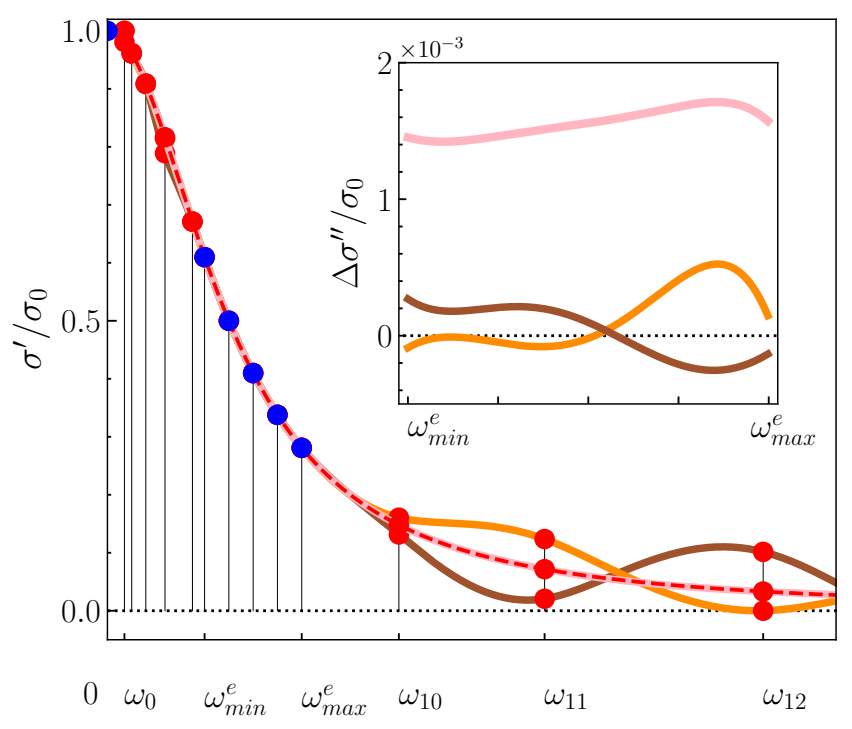

FIG. 1: Reconstruction of Drude complex conductivity by the described method from precisely known values, shown as blue points. The real part of Drude conductivity is shown by the red dashed line together with three curves, which have considerably different values $y_{i}$ (red points) at higher frequencies. Inset shows the deviation $\Delta \sigma^{\prime \prime} / \sigma_{0}=\mathcal{H}\left[\sigma_{\left\{y_{i}\right\}}^{\prime}\right] / \sigma_{0}-g^{\prime \prime}$ of the imaginary part of conductivity calculated from these curves by Hilbert transform, from the Drude one. The deviation is surprisingly larger for the light pink curve, which has all the red points lying on the real part of Drude conductivity, than for the brown and orange curves oscillating around it. The values of the functional (4) for the light pink curve are $\mathcal{F}_{0}=1.2 \times 10^{-5} \sigma_{0}^{2}$ and for the orange and brown curves are $2.0 \times 10^{-7} \sigma_{0}^{2}$. Nevertheless, the average of brown and orange curves is very close to the Drude one.

This approach leads to the following key observation: Suppose, for a moment, that the values of $\sigma_{e}^{\prime}\left(\omega_{i}^{e}\right)$ and $\sigma_{e}^{\prime \prime}\left(\omega_{i}^{e}\right)$ were not obtained by measurement, but were generated instead by restricting the domain of a dimensionless model function $g^{\prime}(\omega)$ and its Hilbert image $g^{\prime \prime}(\omega)$, i.e. $\sigma_{e}^{\prime}\left(\omega_{i}^{e}\right)=\sigma_{0} g^{\prime}\left(\omega_{i}^{e}\right)$ and $\sigma_{e}^{\prime \prime}\left(\omega_{i}^{e}\right)=\sigma_{0} g^{\prime \prime}\left(\omega_{i}^{e}\right)$. It means that the solution corresponding to the input data $\sigma_{e}^{\prime}\left(\omega_{i}^{e}\right)$ and $\sigma_{e}^{\prime \prime}\left(\omega_{i}^{e}\right)$ is already known (the whole function $\left.g^{\prime}(\omega)\right)$. It is clear that the spline of finite number of points $f_{\left\{y_{i}\right\}}(x)$ can not perfectly recover the function, even if $y_{i}=\sigma_{0} g^{\prime}\left(\omega_{i}\right)$. Slight differences yield small, but nonzero values of the functional (4), denoted by $\mathcal{F}_{0}$. However,by applying the corresponding optimization method, one can find curves with an even lower value of the functional (4), while these curves are significantly different from $g^{\prime}(\omega)$.

This is interpreted as a practical consequence of the ill-posed nature of the problem and in order to regularize it, we add the assumption that the searched function is slowly varying. This can be confirmed by the fact that the relaxation rate $\Gamma$ in highly disordered metals has a large value (for estimation of $\Gamma$ see Appendix D and therefore, their conductivity varies on large scales. [4ti- 
lization of this requirement is based on the following idea: For the sake of simplicity, let $g^{\prime}(\omega)$ be the Lorentzian

$$
g^{\prime}(\omega)=\frac{1}{1+(\omega / \Gamma)^{2}}
$$

shown in Fig. 1 as red dashed line. The choice of input data $\sigma_{e}^{\prime}\left(\omega_{i}^{e}\right)$ and the discretization of the problem are depicted in Fig. 1 as blue and red dots respectively. Let us investigate different solutions with the value of functional (4) smaller or equal to the $\mathcal{F}_{0}$. Such solutions have the following property: if the red point at $\omega_{11}$ is lifted and the red point at $\omega_{12}$ lowered, or vice versa, the deviation of the imaginary conductivity from the Drude one, shown in inset of Fig. 1. changes only very slightly and can even decrease the deviation caused by the spline error. Thus, a solution with the least structure can be obtained by averaging of all generated curves .9115 Curves with energy (4) lower than $\mathcal{F}_{0}$ can be found, utilizing the simulated annealing technique by repeated melting of the system. We suggest inverse logarithmic temperature decay and rather small step in the form of $n$-dimensional Gaussian random variable, recommended for continuous optimization ${ }^{[16}$ Optimized parameters are forced to be non-negative and the upper bound is chosen high enough to include the shape of an expected solution but not too high to make optimization time-consuming. Points in the measured interval, i.e. values $y_{i}=\sigma^{\prime}\left(\omega_{i}\right)$ where $\omega_{i} \in\left[\omega_{\text {min }}^{e}, \omega_{\text {max }}^{e}\right]$ are optimized within intervals $\left[\sigma_{e}^{\prime}\left(\omega_{i}^{e}\right)-\chi, \sigma_{e}^{\prime}\left(\omega_{i}^{e}\right)+\chi\right]$, where $\chi$ is the noise level of the data.

The generated curves $\sigma_{\left\{y_{i}\right\}}^{\prime}(\omega)$ which satisfy $\mathcal{F}\left(\sigma_{\left\{y_{i}\right\}}^{\prime}(\omega)\right) \leq \mathcal{F}_{0}$ are used to compute the averaged curve $\overline{\sigma^{\prime}}(\omega)=\sigma_{\left\{\overline{y_{i}}\right\}}^{\prime}(\omega)$. Although the Hilbert transformation is linear, the averaged curve $\overline{\sigma^{\prime}}(\omega)$ gives a slightly higher value of the functional $\mathcal{F}$. Therefore we search a curve varying similarly to $\overline{\sigma^{\prime}}(\omega)$, while minimizing the functional (4). Such a multi-objective optimization is performed by linear scalarization, $\frac{17}{1 . e .}$ minimizing functional:

$$
\tilde{\mathcal{F}}_{\lambda}\left[\sigma_{\left\{y_{i}\right\}}^{\prime}(\omega)\right]=\mathcal{F}\left[\sigma_{\left\{y_{i}\right\}}^{\prime}(\omega)\right]+\lambda \int_{\omega_{0}}^{\omega_{n}}\left(\frac{d^{2} \sigma^{\prime}(\omega)}{d \omega^{2}}-\frac{d^{2} \overline{\sigma^{\prime}}(\omega)}{d \omega^{2}}\right)^{2} \mathrm{~d} \omega,
$$

where $\omega_{0}$ and $\omega_{n}$ are the first and the last point of the spline defined by equation (2). The parameter $\lambda$ is not $a$ priori known and must be carefully chosen for the particular problem. One can start with a high value of $\lambda$, such that $\lambda \gg \mathcal{F} / I$, where $I$ is the integral in (6), estimated with an arbitrarily chosen curve from the ensemble. The found solution is very similar to $\overline{\sigma^{\prime}}(\omega)$ and with a similar value of the first error functional (4). One can lower the value of $\lambda$ further, until the error (4) is lower then $\mathcal{F}_{0}$. In real data analysis, the precision limit $\mathcal{F}_{0}$ is set by the measurement error in such a way, that the accepted curve lies within the estimated measurement error.
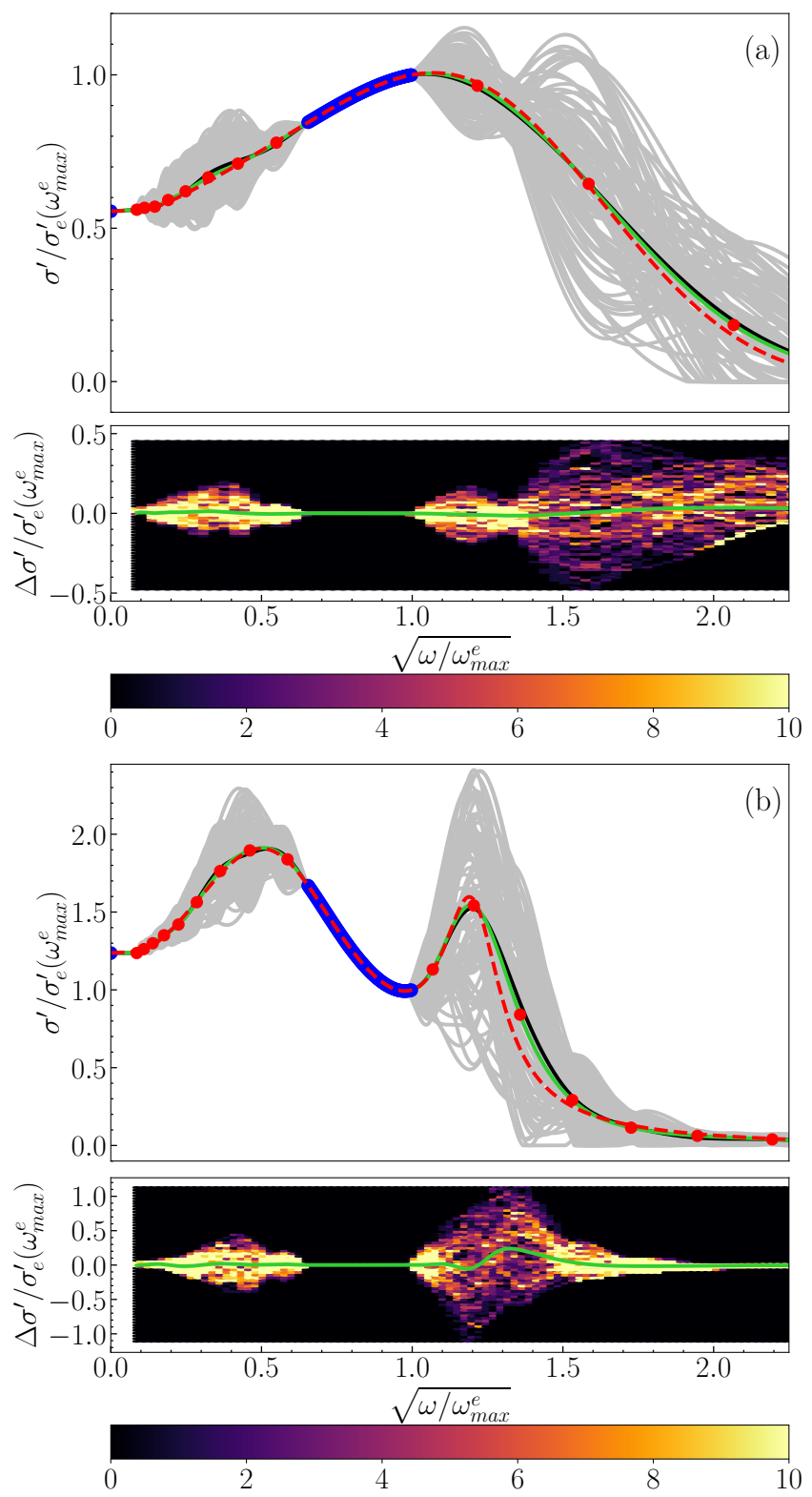

FIG. 2: (a) Top: Red dashed line is a Gaussian model of optical conductivity with square-root quantum corrections (MoC). Blue points laying on the curve are input data for the extrapolation. Silver lines are curves from the ensemble. Black line is a normalized averaged curve $\overline{\sigma^{\prime}}(\omega)=\sigma_{\left\{\overline{y_{i}}\right\}}^{\prime}(\omega)$. The final curve is green. Bottom: Histogram showing occupancy of deviations around the searched function by curves from ensemble and deviation of final curve shown as green line. (b) Top: Red dashed line is the optical conductivity as a sum of two Lorentzians, with square-root quantum corrections $(\mathrm{NbN})$. Blue points are input data, ensemble curves are silver, the average curve is black and the final curve is green. Bottom: histogram of deviation of curves from ensemble and deviation of final green line. 


\section{NUMERICAL RESULTS}

The extrapolation range and accuracy of the described procedure, i. e. finding reasonable complex conductivity curves to extrapolate the experimental curves with respect to KK relations, naturally depends on the degree of complexity of the extrapolated functions. Thus, to demonstrate the feasibility of this method, we tested the extrapolation on sets of data created from two functions, which qualitatively describe the real part of the optical conductivity of $\mathrm{MoC}$ and $\mathrm{NbN}$ thin films, respectively. The conductivity of the former is modeled by a simple function motivated by Ref. 4, which contains the observed square-root quantum corrections to the optical conductivity of these films:

$$
g_{1}(\omega, T)=e^{-\Omega^{2} / \Gamma_{1}^{2}}+Q^{2}\left(\sqrt{\Omega / \Gamma_{1}}-1\right) e^{-4 \Omega^{2} / \Gamma_{1}^{2}} .
$$

Here, $\Omega$ is defined by equation (3), $\Gamma_{1}$ is the scattering rate, and the quantumness $Q^{2}$ characterizes the strength of the square-root corrections, which are significant up to a certain crossover frequency, chosen to be half of the scattering rate. This function is shown as the red dashed line in square-root-scale in top plot of Fig. 2(a) for $Q^{2}=0.66$. The input data - depicted as blue dots - were obtained by sampling the above function (7) at 20 regularly placed frequencies. Points of discretization are depicted as red dots. Next, to test the extrapolation of a slightly more complex function, we study a model function with two peaks motivated by Refs. 34

$$
\begin{aligned}
g_{2}(\omega, T)=\frac{1}{1+\left(\Omega / \Gamma_{2,1}\right)^{2}} & +Q^{2}\left(\sqrt{\Omega / \Gamma_{2,1}}-1\right) e^{-4 \Omega^{2} / \Gamma_{2,1}^{2}} \\
& +\frac{r_{2}}{1+\left(\left(\Omega-\Omega_{2}\right) / \Gamma_{2,2}\right)^{2}},
\end{aligned}
$$

where $\Gamma_{2,1} / 2$ is the cut-off for quantum corrections and $r_{2}$ is the peak height ratio. Here, the cut-off frequency was not determined by the $\Gamma_{2,1}$ but by the appropriate scale describing the ultimate decrease of the function to zero, which we assumed to be $\Omega_{2}+\Gamma_{2,2}$. As we show in the Appendix $D$, the estimated relaxation rate $\Gamma$ for the NbN film corresponds rather to this scale. The searched function (8) is shown in the top plot of Fig. 2(b) as the red dashed line, the input data are blue dots and the optimized points are red. A few curves from the averaged ensemble are also shown as silver lines. The average curves are depicted as black lines and the final conductivity is green. As shown in section (IV), function (8) based on Drude-Lorentz mode ${ }^{3]}$ qualitatively describes the measured conductivity of our NbN films, which indicates the presence of two peaks in optical conductivity.

The results presented in Fig. 2 show the usability of the procedure. For the $g_{1}(\omega)$ model, there is good agreement of the final green curve with the original curve (7) at both higher and lower frequencies. The deviation $\Delta \sigma^{\prime}=g_{1}-\sigma_{y_{i}}^{\prime}$ shown in bottom plot of Fig. 2(a) is below $5 \%$. Shown is also a histogram generated by the ensemble of found curves. The conductivity model $g_{2}(\omega)$ is more complex, therefore, the function (8) is accurately reconstructed in the vicinity of the extrapolated region, whereas further from this region, the deviation increases. The deviation of the final curve from the searched function (8), shown in bottom plot of Fig. 2(b), reaches $20 \%$ and variance of the curves is also larger than for the simpler model (7). This is visible at higher frequencies in the slightly underestimated second peak's height, whereas at low frequencies, the function is recovered with lower deviation. In both cases, we performed both the Padé approx. and SVD. The results are presented and discussed in Appendix C2.

\section{EXTRAPOLATION OF EXPERIMENTAL DATA}

The procedure was applied to extrapolate the conductivity of disordered thin films, as shown in Fig. 3(a) for MoC and Fig. 3(b) for NbN, respectively. For the details of sample preparation, see Appendix B and Refs. 418. Complex conductivity was determined from spectroscopic ellipsometry, and DC conductivity was measured by the Van der Pauw method. The contribution of interband transitions at higher energies must be taken into account in the imaginary part of the conductivity. Following the KK relations, the contribution from the bound electrons can be expressed as $\sigma_{\text {bound }}^{\prime \prime}=-\epsilon_{0}\left(\epsilon_{\infty}-1\right) \omega$, where $\epsilon_{0}$ is the permittivity of vacuum and $\epsilon_{\infty}$ is the bound-electron contribution to the static dielectric constant. For MoC, the value of $\epsilon_{\infty}$ was estimated as 1.4 in Ref. 4 and for $\mathrm{NbN}$, we calculated $\epsilon_{\infty}=2.6$ utilizing the same procedure, while a similar value was estimated in Ref. 3. Subsequently, the corresponding contribution to the imaginary part of the conductivity $\sigma_{\text {bound }}^{\prime \prime}$ was subtracted from measured data. Since the relaxation rate $\Gamma$ can not be easily used as a fitting parameter, we estimated the value from the sheet resistance measured on our thin films and the density of carriers. The details on both estimations of $\epsilon_{\infty}$ and $\Gamma$ are given in the Appendix $\mathrm{D}$. For MoC conductivity, the extrapolation fits between two theoretical curves, a Lorentzian (red) and a Gaussian (pink), proposed in Ref. 4. Taking $\gamma(T)$ in the form $\pi k_{B} T / \hbar$ (same as in Ref. 4), the inverse transformation given by the equation (3) allows to compare the extrapolation (dotted lines) with the terahertz-frequency real part of conductivity (light blue thick lines), obtained from the temperature dependent DC transport measurement. The agreement for MoC is very good, especially taking into account the large distance between optical frequencies $(500 \mathrm{THz})$ and the frequency $(\pi k T / h \approx 50 \mathrm{THz})$ relevant for transport measurements. The agreement for $\mathrm{NbN}$ is less satisfying. However, even for these more complicated conductivity spectra, the extrapolation can match the transport measurements reasonably.

In order to verify the extrapolation procedure experimentally, the real part of conductivity, determined from the extrapolation of ellipsometric data, was compared to 

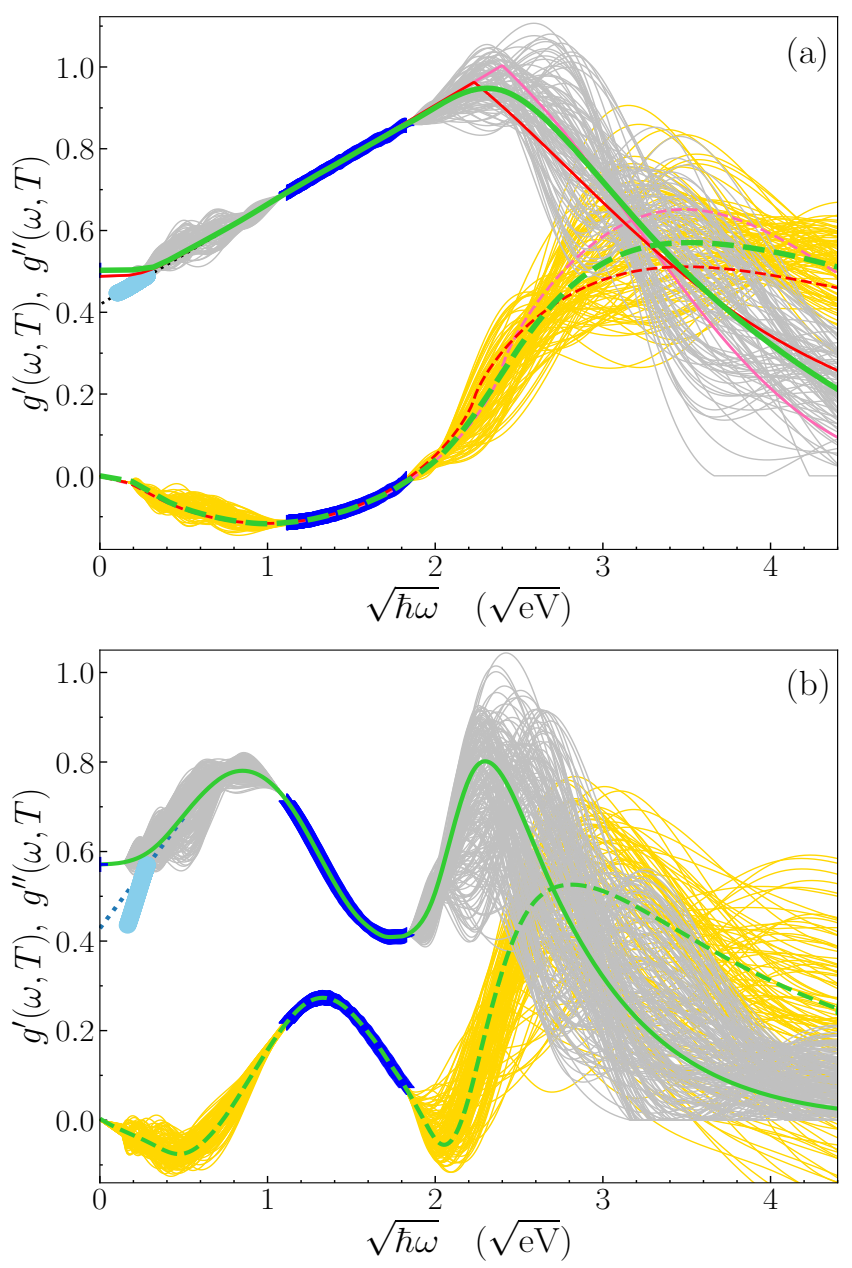

FIG. 3: Real (solid green line) and imaginary (dashed green line) parts of the extrapolation of normalized complex sheet conductance $g=g^{\prime}+i g^{\prime \prime}=\left(\sigma^{\prime}+i \sigma^{\prime \prime}\right) d Z_{0}$, obtained from spectroscopic ellipsometry (blue data) for $\mathrm{MoC}$ (a) and $\mathrm{NbN}$ (b) thin films with thicknesses $d=5 \mathrm{~nm}$ and $3.5 \mathrm{~nm}$, respectively, measured at room temperature. All found complex conductivities with functional (4) $\leq \mathcal{F}_{0}$ are shown as silver (real part) and gold lines (imaginary part). Since Eq. (3) implies $g^{\prime}(\omega=0, T)=g^{\prime}\left(\omega=\pi k_{B} T, 0\right)$, the real part of complex conductivity at zero temperature (dotted line), obtained from extrapolation procedure, can be compared to temperature-dependent DC transport measurement shown as light blue thick lines. Plot (a) also shows two theoretical curves, a Lorentzian (red) and a Gaussian (pink), proposed in Ref. 4 .

the conductivity calculated from the transmission of $\mathrm{MoC}$ and $\mathrm{NbN}$ films (see Appendix A), directly measured in the much wider frequency range accessible by our spectrometer, as shown in Fig. 4. The good agreement of our extrapolation with the data from transmission measurement for $\mathrm{MoC}$ is the encouraging result. The mismatch in the NbN's transmission above $5.0 \mathrm{eV}$ could be caused by interdiffusion between sapphire and $\mathrm{NbN}$. The transmission of sapphire is nearly frequency independent between 0.3-5.0 eV (inset in Fig. 4 (b)), but above $5.0 \mathrm{eV}$, the transmission strongly depends on impurities in the sapphire $\frac{19}{19}$ To verify the reliability of transmission data above $5.0 \mathrm{eV}$, we used them to compute the real part of conductivity, taking the imaginary part from ellipsometry for the same sample, and used both as the basis for extrapolating a complex conductivity curve, plotting its real part (Figure 4). Such extrapolation failed if the entire transmission dataset $(6.2 \mathrm{eV})$ was used, indicating that such conductivity violates the Kramers-Kronig relations. By the bisection method, we found the cut-off, for which the transmission data still process into valid complex conductivity, to be $5.4 \mathrm{eV}$ (dotted line, brown); if a data set with cut-off at $5.0 \mathrm{eV}$ was used, the real part of the generated conductivity (orange) matches that produced from ellipsometry alone (green).

\section{CONCLUSION}

A numerical procedure of extrapolating the complex conductivity of disordered metals based on the KramersKronig relations was introduced. In general, this task has many solutions with standard methods finding a single particular one. The proposed method finds a single solution by averaging many of them which is reasonable, if the requirement of slow variation of their conductivity on energy scale $\lesssim \Gamma$ is met. A slow variation of $\sigma(\omega)$ is reasonably satisfied in highly disordered metals, where experimental data do not indicate rapid oscillations in conductivity and the described method offers a robust and reliable extrapolation procedure of the measured optical conductivity. The range and precision of the extrapolation depend on the complexity of the function. For the simple single-peak model of conductivity, which is the case for MoC thin films, even the DC transport measurements can be reconstructed from the optical measurements, despite the presence of strong quantum corrections. For the more complex, double-peaked conductivity model, which is the case of NbN films, the reliability range of the extrapolation is decreased. However, the extrapolation still reasonably matches the DC measurements and predicts the second peak.

\section{A. Acknowledgments}

This work was supported by the Slovak Research and Development Agency under the contract APVV-16-0372, APVV-18-0358 and by the QuantERA grant SiUCs, by SAS-MTVS. 

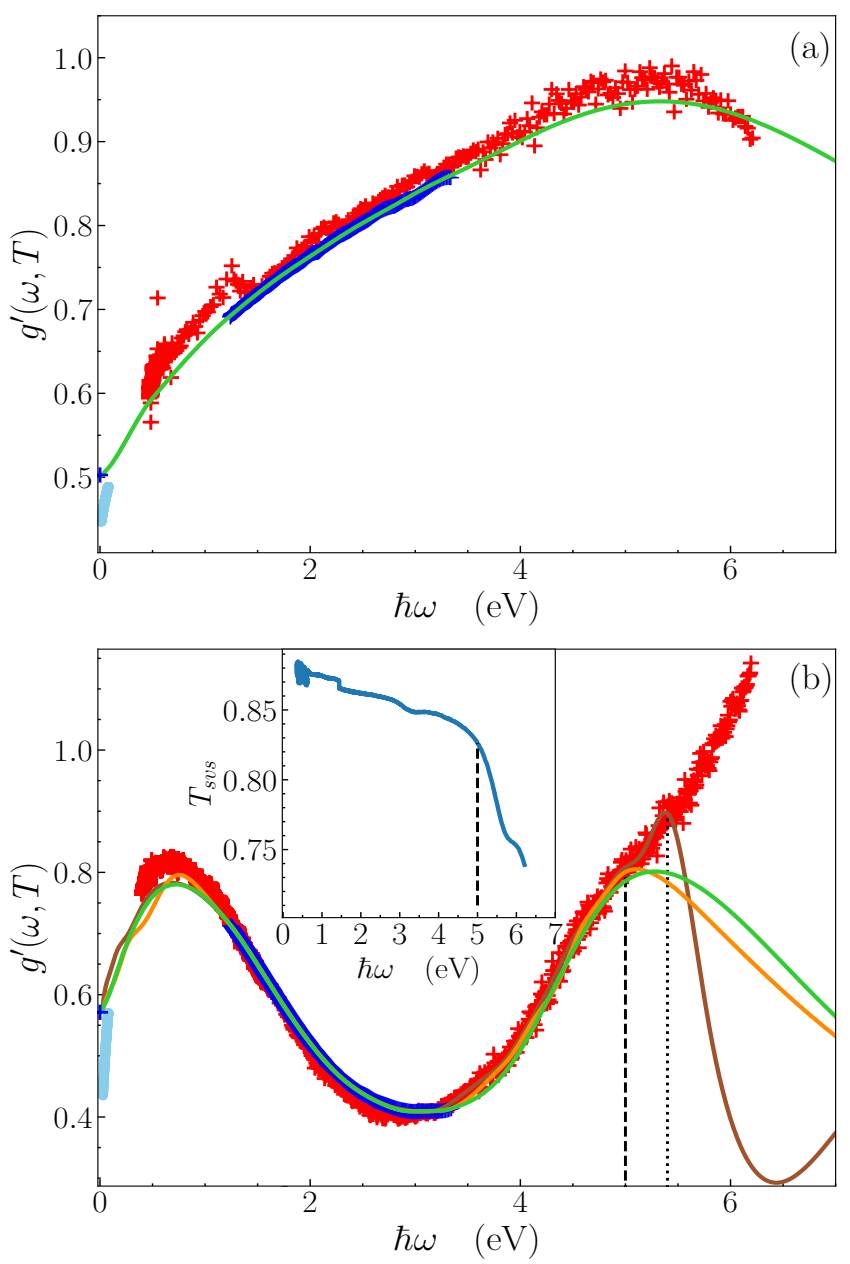

FIG. 4: Green curves are extrapolations of normalized sheet conductance for MoC (a) and NbN (b) obtained from spectroscopic ellipsometry data (blue points) (the same as in Fig. 3) and the experimental values obtained from transport (light blue) and transmission (red) measurements. In plot (b) brown and orange curves are extrapolations of data obtained from transmission measurement with upper cut-off $5.4 \mathrm{eV}$ and $5.0 \mathrm{eV}$, respectively. The value $5.4 \mathrm{eV}$ of the energy cut-off (dotted line) is the largest, where the extrapolation can be done. The highest value of the cut-off energy, where the influence of the substrate can be neglected, is $5.0 \mathrm{eV}$. The rapid change in the brown curve above $5.0 \mathrm{eV}$ is probably due to the influence of the substrate, whose transmission changes rapidly above this value, as shown in the inset. Data are shown in linear scale.

\section{Appendix A: Optical properties calculation}

Both the ellipsometry data and the extrapolated conductivity were used to obtain the optical properties of our thin films. The real and imaginary part of the complex refractive index are defined in Ref. 20 as

$$
\begin{aligned}
& n(\omega)=\sqrt{\frac{1}{2}\left(\Re\left\{\epsilon_{R}(\omega)\right\}+\left|\epsilon_{R}(\omega)\right|\right)} \\
& k(\omega)=\operatorname{sgn}(\omega) \sqrt{\frac{1}{2}\left(-\Re\left\{\epsilon_{R}(\omega)\right\}+\left|\epsilon_{R}(\omega)\right|\right)},
\end{aligned}
$$

where $\epsilon_{R}$ is the (complex) relative permittivity given by

$$
\epsilon_{R}(\omega)=1+i \sigma(\omega) / \omega \epsilon_{0} .
$$

The transmission $T_{v f s}$ of a thin film with the complex refractive index $\tilde{n}(\omega)=n(\omega)+i k(\omega)$, placed upon a semiinfinite substrate with index $\tilde{n}_{s}=n_{s}$, i.e. vacuum-filmsubstrate, whose imaginary part is neglected is 21

$$
T_{v f s}=\left|\frac{2}{\left(1+\tilde{n}_{s}\right) \cos \phi-i\left(\tilde{n}_{s}+\tilde{n}^{2}\right) \phi_{0} \frac{\sin \phi}{\phi}} e^{-i \phi_{0}}\right|^{2} .
$$

Here $\phi=\tilde{n} \frac{\omega}{c} d$ and $\phi_{0}=\frac{\omega}{c} d$ are phase shifts in the film and in vacuum of thicknesses $d$, respectively. The transmission $T_{v f s v}$ through a system with finite substrate thickness is

$$
T_{v f s v}=\frac{T_{v f s} T_{s v}}{1-R_{v f s} R_{s v}},
$$

where $T_{s v}=4 n_{s} /\left(1+n_{s}\right)^{2}$ is the transmission of the substrate-vacuum interface, and the reflection $R$ is obtained from the corresponding transmission simply by $1-T$. Next, the transmission of the vacuum-substratevacuum system is

$$
T_{v s v}=\frac{T_{s v}^{2}}{1-R_{s v}^{2}} .
$$

Finally, the equations A4 and A5 are used to express the transmission of the system vacuum-film-substratevacuum normalized to the transmission of the substrate itself as

$$
\frac{T_{v f s v}}{T_{v s v}}=\frac{T_{v f s}}{T_{s v}} \frac{1-R_{v s}^{2}}{1-R_{v f s} R_{s v}},
$$

which was also a measured quantity. The right-hand side of equation A6 is a function of complex conductivity marked as $T_{n}\left(\sigma^{\prime}, \sigma^{\prime \prime}\right)$ and can be utilized to calculate one of its parts, real or imaginary, from another part and from normalized transmission. Instead of inverting equations A1 - A6 one can simply minimize the difference between $T_{n}\left(\sigma^{\prime}, \sigma^{\prime \prime}\right)$ and measured $T_{v f s v} / T_{v s v}$, namely

$$
\sigma^{\prime}=\arg \min \left(\left(T_{n}\left(\sigma^{\prime}, \sigma^{\prime \prime}\right)-T_{v f s v} / T_{v s v}\right)^{2}\right),
$$

which is, in fact, plotted in Fig. (4) as red symbols.

\section{Appendix B: Sample preparation}

The MoC thin film was prepared by means of reactive magnetron sputtering from Mo target in argonacetylene atmosphere (both gases used of purity 5.0) on 
c-cut sapphire wafer heated to 200 degrees Celsius. The flow rates of argon and acetylene were set by flow meters. During deposition, the magnetron current was held constant at $200 \mathrm{~mA}$, corresponding to a deposition rate $\approx 11 \mathrm{~nm} / \mathrm{min}$. The deposition time, and thus the thickness, was regulated by means of a programmable shutter control interface with a precision of $1 \mathrm{~s}$. The chamber was evacuated to approximately $5 \times 10^{-5} \mathrm{~Pa}$. For details on the preparation of the $\mathrm{MoC}$ films and their characterization see Ref. 22. The sheet resistance of the studied MoC sample with a nominal thickness $d=5 \mathrm{~nm}$ was $R_{\square}=720 \Omega$.

The thin NbN film was prepared by pulsed laser ablation from an Nb-target (purity $99.99 \%$ ) in atmosphere of $\mathrm{N}_{2}$ with added $1 \% \mathrm{H}_{2}$ (purity of the gas mixture is 5.0). The $\mathrm{NbN}$ thin film was deposited on c-cut sapphire wafer, heated to the $600{ }^{\circ} \mathrm{C}$. The used laser fluency of the $\mathrm{KrF}$ laser of $4.9 \mathrm{Jcm}^{-2}$ corresponded to the deposition rate of $2.4 \mathrm{~nm} / \mathrm{min}$. The vacuum chamber was evacuated to $2 \cdot 10^{-6} \mathrm{~Pa}$ before deposition. For more details of the preparation of $\mathrm{NbN}$ film and its growing features see 18 The sheet resistance of our $3.5 \mathrm{~nm}$ thick NbN films was $R_{\square}=655 \Omega$.

\section{Appendix C: Standard methods of extrapolation}

In the following section, the standard methods of the complex conductivity extrapolation are applied to the $\mathrm{MoC}$ and $\mathrm{NbN}$ noisy experimental data and compared to our method.

\section{Fitting to model function}

The presumed model determines a direct physical interpretation of the parameters of the fit, which have a direct physical interpretation, are determined by . The ill-posed nature of the analytic continuation enables one to fit the finite interval data with reasonable accuracy by a variety of models. Therefore, the final solution should be verified by an independent measurements. For example, the conductivity of metallic thin films is commonly analysed by the Drude-Lorentz model. $\frac{23}{\text {, }}$ as presented by Semenov et al. in Ref. 3 for $\mathrm{NbN}$ thin film:

$$
\epsilon_{r}=\epsilon_{\infty}-\frac{\omega_{p}^{2}}{\omega(\omega+i \Gamma)}+\sum_{n=1}^{2} \frac{\Omega_{S n}^{2}}{\Omega_{0 n}^{2}-\omega^{2}-i \Omega_{D n}}
$$

Here, $\epsilon_{r}$ is the relative permittivity from which the conductivity is obtained by equation A2. This function does not take into consideration the presence of quantum corrections to the optical conductivity, which are clearly present in highly disordered NbN films. These corrections can strongly affects the optical conductivity of these films, depending on the degree of disorder. The contribution of quantum corrections to optical conductivity should be present up to UV frequencies, as assumed

\begin{tabular}{|l|l|l|l|l|l|l|l|}
\hline$\omega_{p} \hbar$ & $\Gamma \hbar$ & $\hbar \Omega_{S 1}$ & $\hbar \Omega_{01}$ & $\hbar \Omega_{D 1}$ & $\hbar \Omega_{S 2}$ & $\hbar \Omega_{02}$ & $\hbar \Omega_{D 2}$ \\
\hline \multicolumn{7}{|c|}{$(\mathrm{eV})$} \\
\hline 2.88 & 0.25 & 9.42 & 0.86 & 2.12 & 11.71 & 5.54 & 3.31 \\
\hline
\end{tabular}

TABLE I: Parameters given by the fit of the real and imaginary part of the complex conductivity obtained from spectroscopic ellipsometry on our $\mathrm{NbN}$ sample by model (C1). We assumed $\epsilon_{\infty}=2.6$, coming from calculation as mentioned in the main text.

in theory and recently experimentally demonstrated in Ref. 4. Still, such contributions are usually neglected above infra-red frequencies and the complex conductivity is commonly analysed by the Drude-Lorentz model. This leads to either unrealistic physical parameters or to the conclusion that the complex conductivity cannot be fitted by the standard model ${ }^{24}$ The fit of our NbN data by the Drude-Lorentz model (C1) is shown in Fig. 5. The first Lorentz peak, $i=1$ in (C1), which should corresponds to a band transition, is misused to fit the conductivity suppressed by quantum corrections at lower frequencies and the normal regime at higher frequencies. As a consequence, the Drude contribution is suppressed to create a crossover to the low frequency conductivity and the fit provides an extremely small value of the relaxation rate $\hbar \Gamma \approx 0.26 \mathrm{eV}$ in comparison with rough estimation $\hbar \Gamma \approx 10 \mathrm{eV}$ presented in next section. In fact, all quantities the listed in Tab I are unphysical, as was explicitly pointed out in Ref. 3, too. Moreover, fitting data by an inappropriate physical model can lead to the appearance of additional features that do not have to be real. On the contrary, our "unbiased" method has predictive strength and can uncover phenomena present at frequencies outside the measured interval, just assuming that the optical conductivity cannot rapidly oscillate on a scale determined by the relaxation rate $\Gamma$, whereas rapid changes are not excluded. This is shown in Fig. 5 by arrows which mark frequencies, where optical conductivity changes its monotonicity. Due to quantum corrections, the change can be very sharp, e.g. thermally smeared square-root or logarithmic singularity at zero frequency.

\section{Padé approximation and SVD}

In the Padé approximation, the data are fitted by the ratio of two general complex polynomials, $\sigma(\omega)=$ $P(\omega) / Q(\omega)$ with orders $N$ and $M$, respectively. The first boundary condition is satisfied by including the point at zero frequency and the asymptotic behaviour at higher frequencies is ensured by setting $M=N+1.9$ Results of such constrained Padé approx. strongly depends on the orders $N$ and $M$, which were chosen to avoid reaching negative, unphysical values. This approach offered poor precision of extrapolation (dash-dotted line in Fig. 6) even for the best fit, which was obtained for the orders $N=5$ and $M=6$ for model (7) and $N=4$ and $M=5$ 


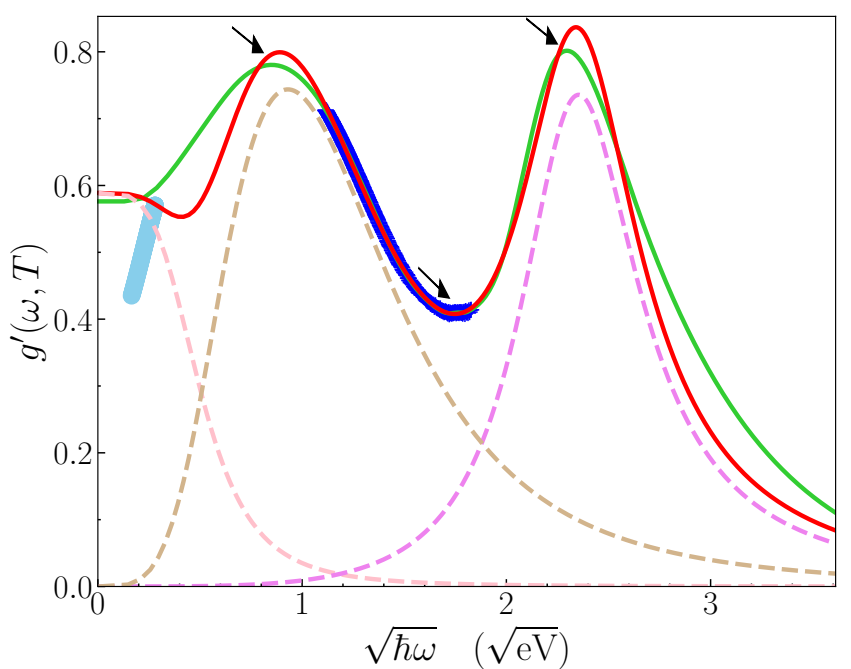

FIG. 5: Fit of the normalized sheet conductance (blue dots), by the Drude-Lorentz model (red line). The fit does not match the increase of the conductivity indicated by the temperaturedependent DC transport measurement (light blue thick line) in contrast to the prolongation (green line), see Fig. 4 (b) in the main text. The pink, brown and violet dashed lines show the contributions from the Drude and the two Lorentz terms in (C1), respectively.

for model (8). Surprisingly, fitting data in the interval $\left[\omega_{\min }^{e}, \omega_{\max }^{e}\right]$ only (solid orange curve), without condition on the orders, offered the best result. For the single peak model (7) the maximum deviation reached was $1 \%$. For the double peak function (8), the result was similar but only in higher frequencies. Non-negativity was again ensured by the orders $N$ and $M$, which were optimized to reach the best match to the value at zero frequency. For the unconstrained Padé, they were both equal to 6 . The Padé approx. which worked reliably for the noiseless model data, was applied to the MoC and NbN noisy experimental data as well (Fig. 7). Although the noise was carefully filtered out, the unconstrained Padé approx. (solid orange curve) rapidly varies and diverges for the $\mathrm{NbN}$ data. The orders of polynomials were chosen to reach the best match to the static conductivity, being $N=5, M=6$ and $N=8, M=9$ for MoC and NbN, respectively. The constrained Padé (dash-dotted line) gave a solution which was more stable, but poorly fitted the data, especially at the edges of the experimental window.

In SVD, the behaviour of the searched function is prescribed at both boundaries. Following Ref. 25, the problem of finding a function satisfying the KK relations, while fitting the input data, is turned into solving a linear system by expanding the known and searched functions into a proper series. Regularization is done by truncating the series and the resulting system is solved by the SVD, which means that the solution is one with the least squares error. For the model function (7), a result similar to Padé was achieved by SVD, with the rank of the decomposed matrix being equal to 7 . The rank was cho-
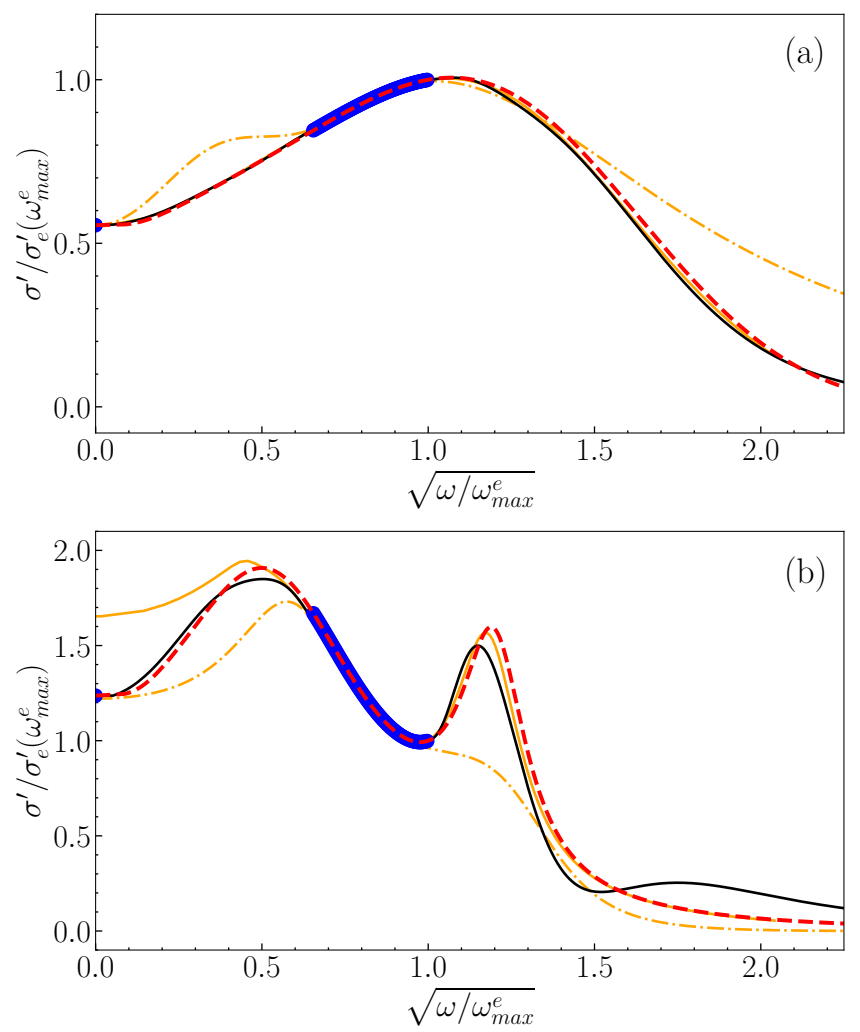

FIG. 6: Application of the Padé and SVD prolongation method on the model data (blue dots, same as in Fig. 22. The red dashed line in panel (a) and (b) corresponds to the model function (7) and (8), respectively. Black line is real part of the solution obtained by SVD, orange dash-dotted line is Padé approx. with both boundary conditions included and orange solid line is Padé approx. without them.

sen to minimize the rapid oscillations of the resulting curve. For the double peak model (8), SVD recovers the searched function at low frequencies very well, but at high frequencies gives additional structure. To obtain this result the matrix rank was equal to 8 . The results obtained from SVD are shown in Fig. 6 as black lines.

When applied to the experimental data, the SVD solution oscillates, its real part reaching negative values, which can not be forbidden in the SVD approach. The rank of the matrix of which we calculated the pseudoinverse by SVD was again chosen to minimize the oscillations, even though they are still present in the final curves (black lines in Fig. 7). This rank was 7 and 6 for $\mathrm{MoC}$ and $\mathrm{NbN}$, respectively.

We believe, that the local variability of the curves generated by our method allows us to suppress the effects of the noise and averaging inside the measured interval provides additional smoothing of data, but with respect to KK. 

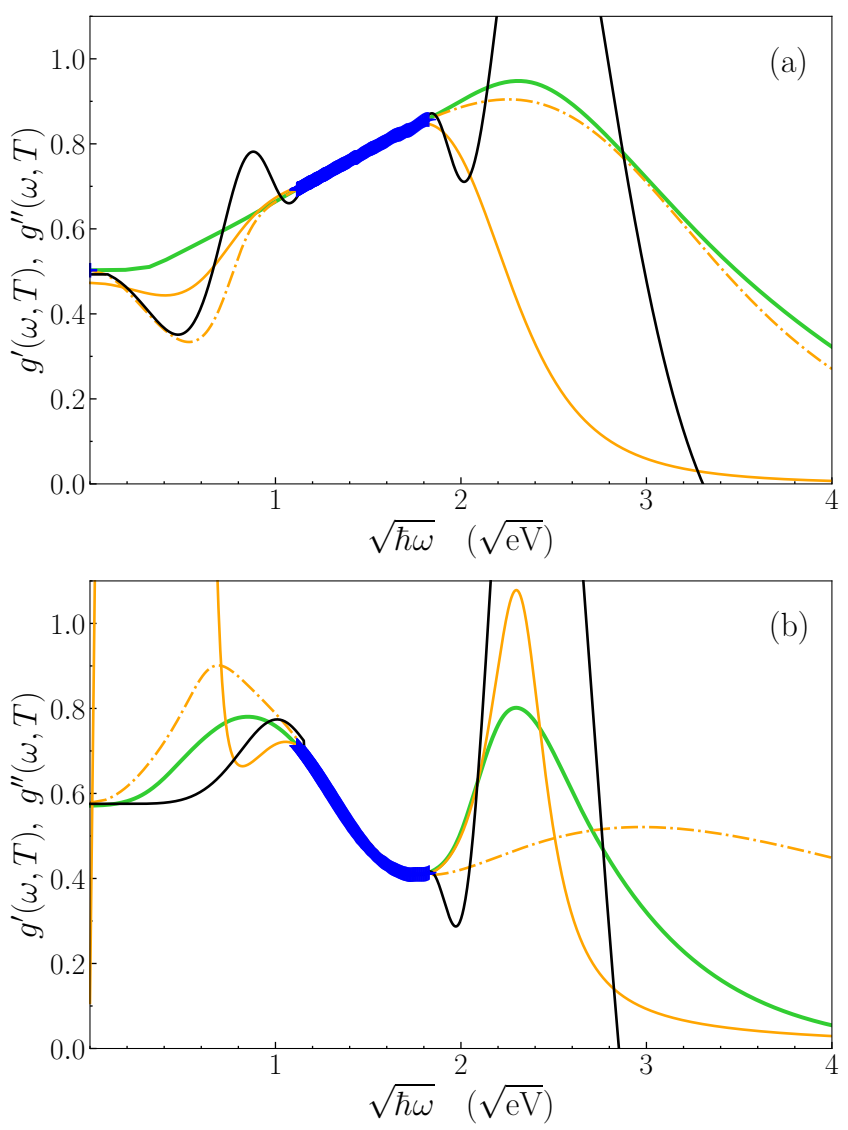

FIG. 7: Comparison of the real parts of normalized sheet conductance obtained by our method (green line) extrapolating ellipsometric (blue) data to the solutions obtained by the Padé approx. (orange solid), constrained Padé (orange dash-dotted) and SVD (black).

\section{Appendix D: Estimation of dielectric constant $\epsilon_{\infty}$ and relaxation rate $\Gamma$}

Extrapolating the complex conductivity as described requires two crucial parameters, electron relaxation rate $\Gamma$ and an "infinity frequency" dielectric constant $\epsilon_{\infty}$. Our estimation of $\epsilon_{\infty}$ closely follows calculation presented in
Ref. 4:

$$
\epsilon_{\infty} \approx 1+\sum \frac{\Omega_{j}^{2}}{\omega_{j}^{2}}
$$

Here, approximate equality means that the high frequency permittivity consists of resonant contributions of transitions between bands modeled by atomic-like levels and conduction band at the Fermi energy. Estimation of weights of the oscillator was based on the $f$-sum rule as $\Omega_{j}^{2}=\frac{Z_{j} n_{a t} e^{2}}{m \epsilon_{0}}$, where $Z_{j}$ is the number of electrons at the atomic level $j$ and $n_{a t}$ is the density of the particular atom type. The excitation energies of the atomic levels $\hbar \omega_{j}$ were taken from Ref. 26 and corresponding densities were calculated from volumes of elementary cells, which can be found in Ref. [27/28. The calculation for MoC yields $\epsilon_{\infty}=1.38{ }^{4}$ Relevant levels of niobium are $4 \mathrm{p}(6$ electrons, $31 \mathrm{eV}), 4 \mathrm{~s}(2$ electrons. $56 \mathrm{eV})$ and $3 \mathrm{~d}(10$ electrons, $203 \mathrm{eV}$ ) and levels of nitrogen are 2s (2electrons, $20 \mathrm{eV}$ ) and $1 \mathrm{~s}$ (2 electrons, $410 \mathrm{eV}$ ) which yields $\epsilon_{\infty}=2.58$ for $\mathrm{NbN}$.

Our rough estimation of the relaxation rate $\Gamma$ is based on the Drude formula for static conductivity

$$
\Gamma=\frac{e^{2} n}{m \sigma_{0}} .
$$

Here, the density of carriers $n \approx 4.2 \times 10^{23} \mathrm{~cm}^{-3}$ is taken from the Hall measurement in Ref. 29. The quantum corrections to the static conductivity can be included via the quantumness $Q^{2} \approx 1 /\left(k_{F} l\right)^{2}$, which relates the classical value $\sigma_{0}$ to the measured one as $\sigma_{d c}=\sigma_{0}\left(1-Q^{2}\right)$ ! For a reasonable quantumness $Q^{2}=0.7$ we obtained $\hbar \Gamma \approx 16 \mathrm{eV}$ and $\approx 10 \mathrm{eV}$ for $\mathrm{MoC}$ and $\mathrm{NbN}$ thin film, respectively. Here we should emphasize, that our method requires a rough estimation of the relaxation time (order of magnitude) only. For example, if $\Gamma$ is overestimated, the method sets more high frequency points close to zero conductivity. On the other hand, if $\Gamma$ is considerably underestimated, the simulated annealing finds no curve. In each case, one can properly adjust $\Gamma$ to obtain reasonable curves found by the simulated annealing.
1 P. K. Day, H. G. LeDuc, B. A. Mazin, A. Vayonakis, and J. Zmuidzinas, Nature 425, 817 (2003).

2 A. Banerjee, R. M. Heath, D. Morozov, D. Hemakumara, U. Nasti, I. Thayne, and R. H. Hadfield, Optical Materials Express 8, 2072 (2018).

3 A. Semenov, B. Günther, U. Böttger, H.-W. Hübers, H. Bartolf, A. Engel, A. Schilling, K. Ilin, M. Siegel, R. Schneider, et al., Physical Review B 80, 054510 (2009).

${ }^{4}$ P. Neilinger, J. Greguš, D. Manca, B. Grančič, M. Kopčík, P. Szabó, P. Samuely, R. Hlubina, and M. Grajcar, Physical Review B 100, 241106(R) (2019).

${ }^{5}$ L. N. Trefethen, BIT Numerical Mathematics pp. 1-15
(2020)

${ }^{6}$ A. Kuzmenko, Review of scientific instruments 76, 083108 (2005).

7 D. B. Tanner, Physical Review B 91, 035123 (2015).

8 B. Altshuler and A. Aronov, in Electron-Electron Interactions in Disordered Systems, edited by A. Efros and M. Pollak (North Holland, 1985).

9 J. Schött, I. L. M. Locht, E. Lundin, O. Grånäs, O. Eriksson, and I. Di Marco, Physical Review B 93, 075104 (2016).

${ }^{10}$ K. S. D. Beach, R. J. Gooding, and F. Marsiglio, Physical Review B 61, 5147 (2000).

11 C. E. Creffield, E. G. Klepfish, E. R. Pike and S. Sarkar 
Phys. Rev. Lett 75, 517-520 (1995)

12 N. Dyn and D. Levin, SIAM Journal on Numerical Analysis 20, 377 (1983).

13 J. Weber, T. Hansen, M. Van de Sanden, and R. Engeln, Journal of Applied Physics 106, 123503 (2009).

14 A. Iske, (2000),URL citeseerx.ist.psu.edu/viewdoc/ download?doi=10.1.1.37.7674\&rep=rep1\&type=pdf

I5 O. Gunnarsson, M. W. Haverkort and G. Sangiovanni, Physical Review B 82, 165125 (2010).

16 J. C. Spall, Introduction to stochastic search and optimization: estimation, simulation, and control, vol. 65 (John Wiley \& Sons, 2005).

17 M. T. Emmerich and A. H. Deutz, Natural computing 17, 585 (2018).

18 S. Volkov, M. Gregor, T. Roch, L. Satrapinskyy, B. Grančič, T. Fiantok, and A. Plecenik, Journal of Electrical Engineering 70, 89 (2019).

19 E. R. Dobrovinskaya, L. A. Lytvynov, and V. Pishchik, Sapphire: material, manufacturing, applications (Springer Science \& Business Media, 2009).

20 E. Hecht, Optics-Addison (Wesley, 2002).

${ }^{21}$ Y. Li and T. F. Heinz, arXiv preprint arXiv:1801.00402 (2018).
22 M. Trgala, M. Žemlička, P. Neilinger, M. Rehák, M. Leporis, Š. Gaži, J. Greguš, T. Plecenik, T. Roch, E. Dobročka, et al., Applied surface science 312, 216 (2014).

23 J. B. Ketterson, The Physics of solids (Oxford University Press, 2016), chap. Metals and the Drude-Lorentz Model.

${ }^{24}$ L. E. Archer, Master's thesis, Massachusetts Institute of Technology, Department of Physics, http://hdl.handle.net/1721.1/112044, unpublished (2017).

25 A. Dienstfrey and L. Greengard, Inverse Problems 17, 1307 (2001).

26 G. P. Williams, Electron binding energies for the elements (2013), URL https://userweb.jlab.org/ gwyn/ ElectronBindingChart2013_D3.pdf

${ }^{27} \mathrm{~K}$. Persson, Materials data on nbn (sg:187) by materials project, (2016), URL https://materialproject.org/ materials/mp-2634/

${ }^{28}$ K. Persson, Materials data on moc (sg:225) by materials project, (2016), URL https://materialproject, org/ metarials/mp-2746/, (2016).

${ }^{29}$ D. Destraz, K. Ilin, M. Siegel, A. Schilling, and J. Chang, Physical Review B 95, 224501 (2017). 